Original Research Article

\title{
Comparative effect of vildagliptin and teneligliptin on HbA1c, glycemic efficacy and insulin sensitivity
}

\author{
Tushar B. Chudiwal*
}

Department of Pharmacology, Ananta Institute of Medical Science and Research Centre, Rajsamand, Rajasthan, India

Received: 23 April 2017

Revised: 16 May 2017

Accepted: 19 May 2017

\section{*Correspondence to:}

Dr. Tushar B. Chudiwal, Email: tusharchudiwal@ gmail.com

Copyright: (C) the author(s), publisher and licensee Medip Academy. This is an openaccess article distributed under the terms of the Creative Commons Attribution NonCommercial License, which permits unrestricted noncommercial use, distribution, and reproduction in any medium, provided the original work is properly cited.

\begin{abstract}
Background: Vildagliptin, a potent and selective dipeptidyl peptidase-4 (DPP4) inhibitor, increases the availability of endogenous incretin hormones, glucagon-like peptide (GLP-1), and glucose-dependent insulinotropic polypeptide, thereby improving glycemic control. The objective of this study is to further investigate the insulin sensitizing properties of Vildagliptin in comparison to those of Teneligliptin.

Methods: Naive subjects with T2DM were administered 50-100mg/day Vildagliptin monotherapy $(\mathrm{n}=53)$. As a comparator, monotherapy, 20mg/day Teneligliptin monotherapy was performed in a non-randomized manner $(n=58)$. No other drugs were administered. At 3 month, levels of diabetic parameters were compared with those at baseline.

Results: At 3 months, while similar reductions of glycated hemoglobin (HbA1c) levels were observed with these two drugs, indexes for insulin sensitivity homeostasis model assessment (HOMA)-R ameliorated only with Vildagliptin. Then, the subjects were divided into two groups representing distinct degrees of insulin resistance; high HOMA-R (C4) and low HOMA-R (2) groups. With Vildagliptin, similar decreases of HbA1c levels were observed in high $(10.85-8.66 \%$, p $\backslash 0.0005)$ and low $(11.12-8.91 \%$, p $\backslash 0.01)$ HOMA-R groups. HOMA-R (-31.9\%, p\0.05) and non-high density lipoprotein cholesterol (non-HDL-C, -7\%, p\0.05) levels significantly decreased. HOMA-B levels increased in both groups with significant inter-group differences $(102.1 \%$ in low HOMA-R group vs. $53.4 \%$ in high HOMA-R group). Group 2. With Teneligliptin similar decreases of HbAlc levels were observed from those of vildagliptin in either high or low HOMA-R group, but no changes of HOMA-R, non-HDL-C levels were noted. Increases of HOMA-B levels with teneligliptin were comparable to those with vildagliptin in either high or low HOMA-R group.

Conclusions: These results indicate that vildagliptin ameliorates insulin sensitivity and non-HDL-C levels in subjects with high degrees of insulin resistance and vildagliptin also shows glycemic efficacy by decreasing HbA1c. This is not the case with teneligliptin though similar glycemic efficacies were observed.
\end{abstract}

Keywords: Glycemic efficacy, HbA1c, Homeostasis model assessment-R, Insulin sensitivity, Teneligliptin, Vildagliptin

\section{INTRODUCTION}

In the management of type 2 diabetes mellitus combinations of oral antidiabetic agents often require achieving good glycemic targets. Nowdays dipeptidyl peptidase-4 (DPP-4) inhibitors is the most suitable agent among non-insulin agents after most widely used
Metformin plus sulfonylurea combination. Vildagliptin, a potent and selective dipeptidyl peptidase-4 inhibitor (DPP-4) inhibitor, increases the availability of endogenous incretin harmones, glucogon - like peptide, and glucose - dependant insulinotropic polypeptide, thereby improving glycemic control. ${ }^{1,2}$ 
As its definition, incretin-based therapies including dipeptidyl peptidase (DPP)-4 inhibitors augment I insulin secretion via the beta-cell activations. ${ }^{3}$ However, their involvement in insulin resistance (or sensitivity), another hallmark for blood glucose regulatory mechanism, remains elusive. Very recently, teneligliptin, a chemotype prolyl-thiazolidine $\neg$ based novel DPP-4 inhibitor, was preliminarily shown to reduce insulin resistance in patients with type 2 diabetes mellitus (T2DM) and in an animal model. ${ }^{4-6}$

Homeostasis model assessment (HOMA)-R and HOMA$\mathrm{B}$ indexes are widely used in the assessment of insulin resistance and beta-cell function, respectively. ${ }^{7}$ They are calculated with insulin and fasting blood glucose (FBG) levels. ${ }^{7}$ However, the usage of these indexes might not be accurate in patients with a low body mass index (BMI), decreased beta-cell cell function and high FBG levels., ${ }^{8,9}$ As a matter of fact, Asian populations including Indians often represent such features. Furthermore, in patients with impaired hepatic and/or renal functions where insulin metabolism may be distorted, HOMA indexes might not be accurate.

In this present work, we further extended the study of insulin sensitizing properties of Vildagliptin. Vildagliptin as a dipeptidyl dipetidase IV inhibitor, in uncontrolled type 2 diabetes mellitus patients who received metformin plus sulfonylyreas significantly improves their glycemic control with less hypoglycemia and no weight gain. ${ }^{10,11}$ Furthermore, it is also proved that Vildagliptin, saxagliptin and sitagliptin as an add therapy in Chinese patients with type 2 diabetes showed similar glycemic control and incidence of adverse effects. ${ }^{12}$ It makes sense to perform this kind of study in drug-naive subjects as monotherapy in order to eliminate the influences of other drugs as much as possible. As an initial step towards investigating this question, vildagliptin 50-100 mg/day monotherapy was administered in newly diagnosed, drugna -ve subjects with T2DM. As a comparator Teneligliptin $20 \mathrm{mg} /$ day monotherapy was performed. Effects, on a number of parameters including insulin resistance and beta-cell function were investigated.

\section{METHODS}

\section{Subjects}

Inclusion criteria were those who were newly diagnosed with T2DM or those who were previously diagnosed but were untreated. The diagnosis was made according to the criteria of ADA. ${ }^{13}$ All the subjects had not received any regularly prescribed drugs in the 6 months prior to the study. Exclusion criteria were those with clinically significant renal [creatinine (CRE) $[1.5 \mathrm{mg} / \mathrm{dL}]$, hepatic [aspartate aminotransferase/alanine aminotransferase (AST/ALT) [70/70IU/L], history of heart disorders, severe hypertension (blood pressure above 160/100mmHg), type 1 diabetes (T1DM) and pregnancy. These subjects were recruited from the outpatient department of Medicine of Ananta Institute of Medical Science and Research Center, Rajsamand. These paitents received either $50-100 \mathrm{mg} /$ day vildagliptin or $20 \mathrm{mg} /$ day teneligliptin monotherapy. Elderly women aged [70years received vildagliptin $50 \mathrm{mg} /$ day. When no improvements of glycemic efficacy were observed, the dose was increased to $100 \mathrm{mg} /$ day. Other subjects received vildagliptin $100 \mathrm{mg} /$ day. At the end of the study, 6 subjects received $50 \mathrm{mg} /$ day and 54 subjects received $100 \mathrm{mg} /$ day. During the study period, the subjects were taking only teneligliptin or vildagliptin and no other medications were administered.

This study was performed in a randomized manner. The subjects were encouraged to follow the exercise and diet suggested by the American Diabetes Association 7. ${ }^{14}$ The protocol was approved by Ethical committee of Ananta Institute of Medical Science and Research, Rajsamand and the informed consent was obtained from the subjects who participated in this study. This study was conducted in accordance with principles of Good Clinical Practice Initially, 60 Subjects from teneligliptin and 60subjects from vildagliptin were enrolled in this project. However, two from teneligliptin group and seven from vildagliptin group had stopped visiting the hospital without giving any reasons. Otherwise, no subjects had dropped out due to intolerance or adverse events. The drop-out subjects were excluded from data analysis.

\section{Laboratory measurements}

The primary end point was the changes in glycated hemoglobin (HbA1c) levels from baseline to 3 months. The HbA1c values were shown with National Glycoprotein Standardization Program (NGSP) standardization throughout this manuscript. ${ }^{15}$ The secondary end point included fasting blood glucose (FBG), insulin, homeostasis model assessment HOMA-R, HOMA-B, body mass index (BMI), total cholesterol (TC), triglycerides (TG), high density lipoprotein (HDL)-C, non-HDL-C and uric acid (UA). Blood was collected at the fasting state before breakfast and the standard technique was used to measure these parameters as described previously. ${ }^{9}$ Measurements of HbA1c and FBG were performed once a month. HOMA-R and HOMA-B, were calculated as described; HOMA-R = insulin 9 FBG/405, HOMA-B = insulin 9 360/(FBG-63). ${ }^{16}$ Hepatic [AST, ALT, alkaline phosphatase (ALP), and gammaglutamyl transpeptidase (GGT)] and renal [blood urea nitrogen (BUN) and CRE] functions were also monitored one month after administration of drugs. In the case of any significant increases of these parameters, administration of drugs was planned to discontinue.

\section{Data analyses}

At 3 months (posttherapy) minus those at baseline (pretherapy), changes in values was calculated. Unpaired Student's 't'test was used to analyze the difference at baseline between the two drug groups, or between these 
two groups representing high (HOMA-R C4) and lovy (HOMA-R12) degree of insulin resistance. ${ }^{16}$ Those HOMA-R falls between 2 and 4 were also analyzed. When the data were normally distributed, paired Student's test was used to analyze the changes in each group (intra group differences). When the data were not normally distributed. Wilcoxon signed-rank test was employed. An analysis of covariance (ANCOVA) was used to analyze the inter-group differences. To identify any factors which influence the changes of HOMA-R levels, multiple regression analyses between the changes of HOMA-R levels as a dependent variable and the baseline levels of other parameters including age, HbA1c, FBG, HOMA-B, BMI, non-HDL-C as independent variables were undertaken. The results were expressed as the mean \pm SD. Throughout the statistical analysis, values of $\mathrm{p} \backslash 0.05$ were considered significant.

\section{RESULTS}

Baseline characteristics of all the subjects and the changes of metabolic parameters with vildagliptin and teneligliptin

The baseline characteristics of all the subjects (Table 1) and the changes of metabolic parameters with 3 month treatment of vildagliptin $50-100 \mathrm{mg} /$ day or teneligliptin $20 \mathrm{mg} /$ day monotherapy (Table 2) are summarized. The baseline characteristics were rather similar in these two drugs. Both HbA1c and FBG levels similarly and effectively decreased with these drugs. HOMA-R levels

had a tendency to decrease only with vildagliptin, while HOMA-B levels similarly, significantly increased with these two drugs. No changes in bodyweight were noted in either drug.

Table 1: Comparison of baseline characteristics of subjects in two groups.

\begin{tabular}{|c|c|c|c|}
\hline Characteristics & Vildagliptin & Teneligliptin & $\begin{array}{l}\text { p } \\
\text { values }\end{array}$ \\
\hline Age (years) & $59.7 \pm 14.4$ & $56.4 \pm 13.6$ & \multirow{6}{*}{ Ns } \\
\hline HbAlc (\%) & $11.10 \pm 2.07$ & $11.11 \pm 2.12$ & \\
\hline FBG (mg/dL) & $205.6 \pm 61.6$ & $215.5 \pm 64.2$ & \\
\hline HOMA-R & $3.56 \pm 3.70$ & $3.56 \pm 2.56$ & \\
\hline HOMA-B & $24.71 \pm 29.05$ & $20.28 \pm 16.19$ & \\
\hline BMI $\left(\mathrm{kg} / \mathrm{m}^{2}\right)$ & $24.73 \pm 4.77$ & $24.56 \pm 4.27$ & \\
\hline
\end{tabular}

-FBG fasting blood glucose, HOMA-R homeostasis model assessment-R, HOMA-B homeostasis model assessment-B, BMI body mass index, ns not significant.

-Values are expressed as mean $\pm \mathrm{SD}$

Table 2: After 3 months' treatment of vildagliptin 50-100mg/day or teneligliptin 20mg/day changes of metabolic parameters.

\begin{tabular}{|c|c|c|c|c|}
\hline Parameter & Baseline & 3 months & $\%$ changes & p values \\
\hline \multicolumn{5}{|l|}{ Vildagliptin } \\
\hline Age (years) & $59.6 \pm 13.4$ & & & \\
\hline HbAlc (\%) & $11.10 \pm 2.07$ & $8.78 \pm 1.99$ & -17.9 & $<0.00001$ \\
\hline FBG $(\mathrm{mg} / \mathrm{dL})$ & $205.6 \pm 61.6$ & $168.0 \pm 64.7$ & -18.3 & $<0.00001$ \\
\hline HOMA-R & $3.56 \pm 3.70$ & $3.27 \pm 2.16$ & -11.9 & 0.075 \\
\hline HOMA-B & $24.71 \pm 29.05$ & $42.42 \pm 41.35$ & 70.9 & $<0.00001$ \\
\hline BMI $\left(\mathrm{kg} / \mathrm{m}^{2}\right)$ & $24.73 \pm 4.77$ & $24.43 \pm 4.38$ & 0.3 & Ns \\
\hline \multicolumn{5}{|l|}{ Teneligliptin } \\
\hline Age (years) & $56.3 \pm 12.6$ & & & \\
\hline HbAlc (\%) & $11.11 \pm 2.12$ & $8.19 \pm 2.12$ & -18.9 & $<0.00001$ \\
\hline $\mathrm{FBG}(\mathrm{mg} / \mathrm{dL})$ & $215.5 \pm 64.2$ & $188.7 \pm 74.2$ & -12 & $<0.0005$ \\
\hline HOMA-R & $3.56 \pm 2.56$ & $3.69 \pm 3.71$ & 4.1 & $\mathrm{Ns}$ \\
\hline HOMA-B & $20.28 \pm 16.19$ & $30.97 \pm 33.13$ & 53.4 & $<0.001$ \\
\hline BMI $\left(\mathrm{kg} / \mathrm{m}^{2}\right)$ & $24.56 \pm 4.27$ & $25.02 \pm 4.27$ & 1 & $\mathrm{Ns}$ \\
\hline
\end{tabular}

Value are expressed as mean \pm SD. The changes of the indicated parameters were compared before and after treatment FBG fasting blood glucose, HOMA-R homeostasis model assessment-R, HOMA-B homeostasis model assessment-B, BMI body mass index

\section{Baseline characteristics of subjects in two groups with distinct degrees of insulin resistance}

The above data indicate that indexes for insulin resistance [HOMA-R] appear to ameliorate with vildagliptin, but not with teneligliptin (Table 2). Then, the subjects were divided into two groups; those with high degrees of insulin resistance (baseline HOMA-R $\mathrm{C} 4, \mathrm{n}=16$ for vildagliptin and $n=25$ for teneligliptin) and others with low degrees of insulin resistance (baseline HOMA-R 2 , $\mathrm{n}=20$ for vildagliptin and $\mathrm{n}=23$ for teneligliptin). At baseline, similar HbA1c and FBG levels were observed in these two sub-groups with vildagliptin and teneligliptin. However, HOMA-B, insulin, and BMI levels were significantly higher in high HOMA-R group 
than low HOMA-R group with both of these drugs. The subjects in high HOMA-R group with teneligliptin appeared to have higher FBG and lower HOMA-B levels in comparison to those with Vildagliptin (Table 3).

\section{Effect of vildagliptin or teneligliptin on diabetic parameters in two groups with distinct degrees of insulin resistance}

At 3 months, HOMA-R levels significantly decreased with vildagliptin in high HOMA-R group, while they had no changes in low HOMA-R group (Table 4).

Insulin and BMI are well known to be associated with insulin resistance. Involvement of non-HDL-C levels in insulin resistance was further investigated. With both drugs, baseline non-HDL-C levels were significantly higher in high HOMA-R group than low HOMA-R group (Table 3). However, significant reductions of non-HDL-C levels were observed only in high HOMA-R group with Vildagliptin (Table 4).

\section{Safety and tolerability}

Two from high HOMA-R group and one from low HOMA-R group with vildagliptin, and four from high HOMA-R group and three from low HOMA-R group with teneligliptin reported mid-hypoglycemic events, which could be easily managed by taking glucose drinks by themselves. These potential adverse events occurred in the first 4 weeks of the initiation of the drug. Otherwise no subjects had any clinically significant elevations of renal or hepatic enzymes and no gastrointestinal complains were observed. No subjects had dropped out because of intolerance or adverse events.

Table 3: Baseline metabolic parameter's comparison in two groups with distinct degrees of insulin resistance.

\begin{tabular}{|c|c|c|c|}
\hline Parameter & $\begin{array}{l}\text { Low } \\
\text { HOMA-R }\end{array}$ & $\begin{array}{l}\text { High } \\
\text { HOMA-R }\end{array}$ & p values \\
\hline \multicolumn{4}{|l|}{ Vildagliptin } \\
\hline Age(years) & $60.0 \pm 12.8$ & $55.8 \pm 13.2$ & Ns \\
\hline HOMA-R & $1.48 \pm 0.48$ & $7.02 \pm 4.79$ & $<0.00001$ \\
\hline HOMA-B & $9.56 \pm 4.58$ & $42.76 \pm 37.69$ & $<0.0005$ \\
\hline $\operatorname{HbAlc}(\%)$ & $11.12 \pm 2.36$ & $10.85 \pm 1.48$ & Ns \\
\hline FBG(mg/dL) & $187.8 \pm 47.9$ & $212.4 \pm 44.1$ & Ns \\
\hline $\mathrm{BM} 1\left(\mathrm{~kg} / \mathrm{m}^{2}\right)$ & $22.47 \pm 2.31$ & $28.59 \pm 5.79$ & $<0.00001$ \\
\hline $\begin{array}{l}\text { Non-HDL-C } \\
(\mathrm{mg} / \mathrm{dL})\end{array}$ & $165.2 \pm 30.4$ & $175.8 \pm 30.8$ & $<0.05$ \\
\hline \multicolumn{4}{|l|}{ Teneligliptin } \\
\hline Age(years) & $57.7 \pm 12.6$ & $54.2 \pm 14.8$ & Ns \\
\hline HOMA-R & $1.73 \pm 1.41$ & $6.51 \pm 2.69$ & $<0.00001$ \\
\hline HOMA-B & $17.93 \pm 16.90$ & $26.64 \pm 18.88$ & $<0.05$ \\
\hline $\mathrm{HbA} 1 \mathrm{c}(\%)$ & $10.44 \pm 2.78$ & $11.68 \pm 1.81$ & Ns \\
\hline $\mathrm{FBG}(\mathrm{mg} / \mathrm{dL})$ & $189.1 \pm 67.3$ & $252.3 \pm 61.6$ & Ns \\
\hline BMI $\left(\mathrm{kg} / \mathrm{m}^{2}\right)$ & $24.28 \pm 4.38$ & $27.94 \pm 4.52$ & $<0.005$ \\
\hline $\begin{array}{l}\text { Non-HDL-C } \\
(\mathrm{mg} / \mathrm{dL})\end{array}$ & $153.5 \pm 29.2$ & $198.1 \pm 47.2$ & $<0.001$ \\
\hline \multicolumn{4}{|c|}{$\begin{array}{l}\text { Value are expressed as mean } \pm \text { SD. The baseline levels of } \\
\text { indicated parameters were compared between low and high } \\
\text { HOMA-R groups FBG fasting blood glucose, HOMA-R } \\
\text { homeostasis model assessment-R, HOMA-B homeostasis model } \\
\text { assessment-B, BMI body mass index, non-HDL-C non-high } \\
\text { density lipoprotein cholesterol }\end{array}$} \\
\hline
\end{tabular}

Table 4: After 3 months' treatment of vildagliptin 50-100mg/day, based on distinct baseline HOMA changes of metabolic parameters.

\begin{tabular}{|c|c|c|c|c|}
\hline Parameter & Baseline & 3 months & $\%$ Changes & P values \\
\hline \multicolumn{5}{|l|}{ High HOMA-R } \\
\hline Age (years) & $55.8 \pm 13.2$ & & & \\
\hline HOMA-R & $7.02 \pm 4.79$ & $4.56 \pm 2.33$ & -31.9 & $<0.05$ \\
\hline HOMA-B & $42.76 \pm 37.69$ & $66.70 \pm 43.39$ & 53.4 & $<0.0005$ \\
\hline HbAlc $(\%)$ & $10.85 \pm 1.48$ & $8.66 \pm 1.41$ & -21.9 & $<0.0005$ \\
\hline $\mathrm{FBG}(\mathrm{mg} / \mathrm{dL})$ & $212.4 \pm 44.1$ & $164.1 \pm 59.4$ & -24.1 & $<0.00001$ \\
\hline BMI $\left(\mathrm{kg} / \mathrm{m}^{2}\right)$ & $28.59 \pm 5.79$ & $27.94 \pm 5.32$ & -1.3 & $\mathrm{Ns}$ \\
\hline Non-HDL-C (mg/dL) & $175.8 \pm 30.8$ & $168.7 \pm 36.2$ & -7 & $<0.05$ \\
\hline \multicolumn{5}{|l|}{ Low HOMA-R } \\
\hline Age (years) & $60.0 \pm 12.8$ & & & \\
\hline HOMA-R & $1.48 \pm 0.48$ & $2.14 \pm 1.69$ & 41.9 & Ns \\
\hline HOMA-B & $9.56 \pm 4.58$ & $18.97 \pm 13.90$ & 102.1 & $<0.005$ \\
\hline HbAlc (\%) & $11.12 \pm 2.36$ & $8.91 \pm 2.19$ & -16.1 & $<0.001$ \\
\hline FBG (mg/dL) & $187.8 \pm 47.9$ & $168.2 \pm 59.7$ & -13.9 & $<0.05$ \\
\hline BMI $\left(\mathrm{kg} / \mathrm{m}^{2}\right)$ & $22.47 \pm 2.31$ & $21.92 \pm 2.61$ & 1.7 & $<0.05$ \\
\hline Non-HDL-C (mg/dL) & $165.2 \pm 30.4$ & $155.2 \pm 43.3$ & 3.1 & Ns \\
\hline
\end{tabular}

Value are expressed as mean \pm SD. The changes of the indicated parameters were compared before and after treatment FBG fasting blood glucose, HOMA-R homeostasis model assessment-R, HOMA-B homeostasis model assessment-B, BMI body mass index, non-HDL-C non- high density lipoprotein cholesterol 
Table 5: After 3 months' treatment of teneligliptin 20mg/day based on distinct baseline HOMA-R levels changes of metabolic parameters.

\begin{tabular}{|c|c|c|c|c|}
\hline Parameter & Baseline & 3 months & $\%$ Changes & p values \\
\hline \multicolumn{5}{|l|}{ High HOMA-R } \\
\hline Age (years) & $54.2 \pm 14.8$ & & & \\
\hline HOMA-R & $6.51 \pm 2.69$ & $6.44 \pm 5.09$ & 0.59 & ns \\
\hline HOMA-B & $26.64 \pm 18.88$ & $33.27 \pm 23.79$ & 28.9 & $<0.05$ \\
\hline $\mathrm{HbA}$ lc $(\%)$ & $11.68 \pm 1.81$ & $9.17 \pm 1.79$ & -18.1 & $<0.001$ \\
\hline FBG (mg/dL) & $252.3 \pm 61.6$ & $221.0 \pm 6.4$ & -12.1 & $<0.05$ \\
\hline BMI $\left(\mathrm{kg} / \mathrm{m}^{2}\right)$ & $27.94 \pm 4.52$ & $27.69 \pm 4.48$ & 0.69 & ns \\
\hline Non-HDL-C (mg/dL) & $198.1 \pm 47.2$ & $189.3 \pm 42.0$ & -4.1 & ns \\
\hline \multicolumn{5}{|l|}{ Low HOMA-R } \\
\hline Age (years) & $57.7 \pm 12.6$ & & & \\
\hline HOMA-R & $1.73 \pm 1.41$ & $2.36 \pm 1.69$ & 31.9 & ns \\
\hline HOMA-B & $17.93 \pm 16.90$ & $35.65 \pm 51.6$ & 108.9 & $<0.05$ \\
\hline HbA lc (\%) & $10.44 \pm 2.78$ & $8.78 \pm 2.37$ & -17.9 & $<0.001$ \\
\hline FGB (mg/dL) & $189.1 \pm 67.3$ & $172.9 \pm 63.8$ & -8.3 & $<0.05$ \\
\hline BMI $\left(\mathrm{kg} / \mathrm{m}^{2}\right)$ & $24.28 \pm 4.38$ & $23.3 \pm 4.57$ & 0.49 & ns \\
\hline Non-HDL-C (mg/dL) & $153.5 \pm 29.2$ & $147.2 \pm 33.9$ & 0.28 & ns \\
\hline
\end{tabular}

Values are expressed as mean \pm SD. The changes of the indicated parameters were compared before and after treatment FBG fasting blood glucose, HOMA-R homeostasis model assessment-R, HOMA-B homeostasis model assessment-B, BMI body mass index, nonHDL-C non-high density lipoprotein cholesterol

\section{DISCUSSION}

\section{Insulin sensitizing properties of vildagliptin and teneligliptin}

Most researchers use HOMA-R index to evaluate the degrees of insulin resistance. It was shown that vildagliptin could ameliorate insulin sensitivity in subjects with high degrees of insulin resistance (Table 4), whereas teneligliptin had no such effects (Table 5).

There are conflicting reports regarding the insulin sensitizing properties of teneligliptin. Some researchers reported beneficial effects, while others reported no such effects with teneligliptin. The following reasons can be postulated to explain this discrepancy. Study showing the beneficial effects of teneligliptin on insulin sensitivity was not with monotherapy. ${ }^{17,18}$ In these papers, the insulin-sensitizing properties of teneligliptin were evaluated as add-on to other diabetes e.g. metformin). A possibility remains that teneligliptin itself has no effect on insulin sensitivity but by lowering the glucose levels with the addition of this drug via the incretin effect, glucotoxicity was ameliorated, thereby improving the degree of insulin resistance (and beta-cell function). In the present study and others that showed no effect teneligliptin on insulin sensitivity. In the papers of Kadowaki T, Kondo K. et al, 40mg OD dose of Teneligliptin was used, where $40 \mathrm{mg}$ OD dose of Teneligliptin showed better results than 20mg OD doses. ${ }^{19,20}$ While in our work, $20 \mathrm{mg}$ of this drug was employed. It can be that insulin-sensitizing properties could be observed with 40mg OD Teneligliptin but not with lower doses.

Insulin resistance is regarded as distorted physiological response to insulin. It is characterized by compensatory hyperinsulinemia and is associated with obesity and pathophysiology of T2DM. ${ }^{21}$ The underlying mechanism of insulin-sensitizing properties of teneligliptin remains to be investigated. One hypothesis is that in addition to inhibiting DPP-4 enzymes thereby elevating incretins, teneligliptin may have effects on other factors that may be responsible in insulin sensitivity (e.g. AMP activated protein kinase. glucose transporter 4). Molecular and cellular approaches are required in order to clarify this issue. It remains to be investigated whether this favourable effect on insulin resistance may lead to reduced risks for cardiovascular disorders and whether or not similar results could be obtained with other DPP-4 inhibitors.

\section{Baseline differences and differential effects of metabolic parameters with vildagliptin depending on the degrees of insulin resistance}

When the metabolic parameters were compared at baseline, insulin, HOMA-B, BMI and non-HDL-C levels were significantly higher in high HOMA-R group than low HOMA-R group with either vildagliptin or teneligliptin (Table 3), indicating that these parameters are associated with the degrees of insulin resistance.

So far, few studies are available on the relationship between DPP-4 inhibitors and atherogenic lipids 
including non-HDL-C. Non-HDL-C (total cholesterol minus HDL-C) provides a convenient measure of the cholesterol content of all atherogenic lipoproteins and it has been preliminarily shown to be elevated in subjects with metabolic syndrome. ${ }^{22}$ This is consistent with this present study showing that nonHDL-C levels are higher in high HOMA-R group than low HOMA-R group (Table 3). Non-HDL-C levels decreased with vildagliptin (but not with teneligliptin) in high HOMA-R group (Table 4), indicating that this drug has favourable outcomes on cardiovascular disorders.

In high HOMA-R group with vildagliptin, higher levels of FBG decreased in comparison to low HOMA-R group (24.1 vs. $-13.9 \%$; inter-group difference 00.05 , Table 4) while HbA1c similarly decreased in these two groups (Table 4). This observation indirectly indicates that the degrees of post-meal glucose reductions with vildagliptin would be greater in low HOMA-R group than high HOMA-R group. Other parameters showed distinct regulatory patterns in these two groups. Briefly, in high HOMA-R group, improvements of insulin resistance assessed by HOMA-R were observed (Table 4). In low HOMA-R group, higher degrees of enhancement of betacell function (HOMA-B) were observed in comparison to high HOMA-R group Nevertheless, similar HbA1 clowering effects were observed in these two groups. These results suggest that glycemic efficacy of vildagliptin may be determined by the balance of its capacity in modulating insulin resistance and beta-cell function depending on the degrees of baseline levels of insulin resistance. In either case, similar HbAlc lowering effects of this drug were observed. Thus, it is beneficial to measure the degree of insulin resistance before starting this drug.

\section{Glycemic efficacy of teneligliptin}

With teneligliptin, indexes for insulin sensitivity [assessed by decreased HOMA-R] had no changes in high HOMA$\mathrm{R}$ group (Tables 2). By contrast, similar, significant increases of beta-cell function (assessed by HOMA-B) were observed in comparison to those of vildagliptin (compare Tables 2 and 4). Teneligliptin have nevertheless similar HbA1c ᄀlowering efficacies from those of vildagliptin which has favourable effects on insulin sensitivity. Vildagliptin has glucose lowering mechanisms that are not present in teneligliptin (or other drugs). This was not observed with teneligliptin (E. Kutoh, unpublished observation).

The results showing that increases of bodyweight in low HOMA-R group with Vildagliptin (Table 4) and that somewhat higher degrees of HbA1c-lowering efficacy in low HOMA-R group with Teneligliptin in comparison to Vildagliptin (Table 4) could be associated with the differential increase of glucose-dependent insulinotropic polypeptide (GIP) and glucagon-like peptide (GLP)-1 with these two drugs. This could also be one of the reasons for the non-inferior glycemic efficacies of teneligliptin even without the effect on insulin sensitivity.

\section{CONCLUSION}

The results of this manuscript add the following novel information to our current knowledge: Vildagliptin has favourable effects on down-regulating high degrees of insulin resistance. This was confirmed using the conventional HOMA-index. However, this is not the case with Teneligliptin though, similar glycemic efficacies were observed. Teneligliptin may have distinct glucoselowering properties from those of vildagliptin. Glycemic efficacy of vildagliptin may be determined by the balance of its capacity in modulating insulin resistance and betacell function depending on the degree of insulin resistance.

\section{Funding: No funding sources}

Conflict of interest: None declared

Ethical approval: The study was approved accordance with the Good Clinical Practice (GCP) guidelines

\section{REFERENCES}

1. Balas B, Baig MR, Watson C, Dunning BE, Ligueros-Saylan M, Wang Y, et al. The dipeptidyl Peptidase IV inhibitor vildagliptin suppresses endogenous glucose production and enhances islet function after single-dose. Administration in type 2 diabetic patients. J Clin Endocrinol Metab. 2007;92:1249-55.

2. Mari A, Sallas WM, He YL, Watson C, LiguerosSaylan M, Dunning BE, et al. Vildagliptin, a dipeptidyl peptidase 4 inhibitor, improves modelassessed beta-cell function in patients with type 2 diabetes. J Clin Endocrinol Metab. 2005;90:4888-94.

3. Harada N, lnagaki N. Incretin and incretin-based therapies. Nihon Rinsho. 2010;68(5):931-42.

4. Nakamaru Y, Hayashi Y, Ikegawa R, Kinoshita S, Madera BP, Gunput D, et al. Metabolism and disposition of the dipeptidyl peptidase IV inhibitor teneligliptin in humans. Xenobiotica. 2014;44(3):242-53.

5. Kadowaki T, Kondo K. Efficacy, safety and doseresponse relationship of teneligliptin, a dipeptidyl peptidase-4 inhibitor, in Japanese patients with type 2 diabetes mellitus. Diabetes Obes Metab. 2013;15(9):810-8.

6. Nakagami H, Pang Z, Shimosato T, Moritani T, Kurinami H, Koriyama $\mathrm{H}$, et al. The dipeptidyl peptidase-4 inhibitor teneligliptin improved endothelial dysfunction and insulin resistance in the SHR/NDmcr-cp rat model of metabolic syndrome. Hypertens Res. 2014;37(7):629-35.

7. Matthews DR, Hosker JP, Rudenski AS, Naylor BA, Treacher DF, Tumer RC. Homeostasis model assessment: insulin resistance and beta-cell function from fasting plasma glucose and insulin 
concentrations in man. Diabetologia. 1985;28(7):4129.

8. Ohkura T, Shiochi H, Fujioka Y, Sumi K, Yamamoto N, Matsuzawa K, et al. 20/ (fasting C-peptide 9 fasting plasma glucose) is a simple and effective index of insulin resistance in patients with type 2 diabetes mellitus: a preliminary report. Cardiovasc Diabetol. 2013;12:21.

9. Kutoh E, Hirate M, Wada A. Distinct glucoselowering properties in good responders treated with sitagliptin and alogliptin. Int $\mathrm{J}$ Clin Pract. 2015;69(11):1296-302.

10. Hong AR, Leeb J, Kuwangbob EJ, Kyoung MK. Comparison of Vildagliptin as an add-on therapy and sulfonylurea dose-increasing therapy in patients with inadequately controlled type 2 diabetes using metformin and sulfonylurea. Diabetes Research and Clinical Practice. July 2015;109(1):141-8.

11. Bosi E. Vildagliptin Plus Metformin Combination therapy provides superior glycemic control to individual monotherapy in treatment-naive patients with type 2 Diabetes Mellitus. Diabetes Obes Metab. 2009; 11:506-15.

12. Chun-Jun L, Xiao-Juan L, Bai L. Efficacy and Safety Of Vildagliptin, Saxagliptin Or Sitagliptin as an addon Therapy in Chinese Patients with type 2 Diabetes, inadequately controlled with a dual combination of traditional oral hypoglycemic agents. Diabetology and Metabolic Syndrome. 2014;6(1).

13. American Diabetes Association. Standards of medical care in diabetes. Diabetes Care 2014;37(1):S14-80.

14. Ma Y, Olendzki BC, Merriam PA, Chiriboga DE, Culver AL, Li W, et al. A randomized clinical trial comparing low-glycemic index versus ADA dietary education among individuals with type 2 diabetes. Nutrition. 2008;24(1):45-56.
15. Miedema K. Towards worldwide standardisation of HbAlc determination. Diabetologia. 2004;47(7):1143-8.

16. Kutoh E, Fukushima T. Insulin-dependent actions of pioglitazone in newly diagnosed, drug naive patients with type 2 diabetes. Endocrine. 2009;35(3):333-40.

17. Tsuchimochi W, Ueno H, Yamashita E, Tsubouchi C, Sakoda H, Nakamura S, et al. Teneligliptin improves glycemic control with the reduction of postprandial insulin requirement in Japanese diabetic patients. Endocr J. 2015;62(1):13-20.

18. Kishimoto M. Teneligliptin: A DPP-4 inhibitor for the treatment of type 2 diabetes. Diabetes Metab Syndr Obes. 2013;6:187-95.

19. Kadowaki T, Kondo K. Efficacy and safety of teneligliptin added to glimepiride in Japanese patients with type 2 diabetes mellitus: A randomized, doubleblind, placebocontrolled study with an open-label, long-term extension. Diabetes Obes Metab. 2014;16;418-25.

20. Kadowaki T, Kondo K. Efficacy and sagety of teneligliptin in combination with pioglitazone in Japanese Patients with type 2 diabetes mellitus. J Diabetes Investig. 2013;4;576-84.

21. Funahashi T, Matsuzawa Y. Obesity and diabetes mellitus. Nihon Naika Gakkai Z Asshi. 1996;85(4):578-82.

22. Liu A, Reaven GM. Is measurement of non-HDL cholesterol an effective way to identify the metabolic syndrome? Nutr Metab Cardiovasc Dis. 2013;23(11)1122-7.

Cite this article as: Chudiwal TB. Comparative effect of vildagliptin and teneligliptin on $\mathrm{HbA1c}$, glycemic efficacy and insulin sensitivity. Int J Basic Clin Pharmacol 2017;6:1682-8. 